\title{
Research of Numerical Simulation of Biomechanics on In-stent-restenosis
}

Aike Qiao*, Yulin Fu and Zhanzhu Zhang

College of Life Science and Bio-engineering, Beijing University of Technology, Beijing, 100124, China

*Corresponding author: Aike Qiao, College of Life Science and Bio-engineering, Beijing University of Technology, Beijing, 100124, China, Tel: 8610-67396657; E-mail: qak@bjut.edu.cn

Rec date: Feb 05, 2015; Acc date: Feb 19, 2015; Pub date: Feb 21, 2015

Copyright: @ 2015 Qiao A, et al. This is an open-access article distributed under the terms of the Creative Commons Attribution License, which permits unrestricted use, distribution, and reproduction in any medium, provided the original author and source are credited.

\begin{abstract}
Stenting intervention is emerging as an alternative for treating the stenosis of cardiac and cerebral arteries. However, postoperative In-Stent-Restenosis (ISR) remains a challenge for medical sciences and biomechanical engineering. ISR is related not only to the stress induced by the mechanical support of stent struts on arterial wall, but also to the intimal hyperplasia induced by the hemodynamic changes. The recent research on biomechanics of stented artery was reviewed in this paper. In particular, from the perspectives of solid mechanics and hemodynamics, the research progress of stented artery using biomechanical simulation was discussed. The biomechanical factors associated with ISR were analyzed and summarized. Numerical simulation is a powerful approach for the investigation of the relationship between stenting intervention and ISR, and can provide scientific guidelines for the design of stent structure and clinical procedure of stenting intervention.
\end{abstract}

Keywords: Arterial stenosis; Hemodynamics; Endovascular stent; In-stent-restenosis; Numerical simulation

\section{Introduction}

In modern society, the increasingly high incidence of cardiovascular and cerebrovascular diseases becomes the first life-threatening culprit to human health and safety. Among these diseases atherosclerosis is so common and popular. Atherosclerosis is a diffuse vascular disease whose effects are potentially additive, eventually induces the arterial stenosis, and is the most important cause of myocardial infarction and ischemic stroke [1]. Currently, treatment of arterial stenosis can be divided into three kinds of procedures, i.e. medical drug, surgical operation and endovascular intervention treatment. Drug treatment, with low effect, long dosing cycle and side effect of drug dependence for patients, can only reduce $13 \%$ of the relative risk [2]. The surgery harm on the patient is permanent, and the ensuing complications and mortality rate are also very high [2]. The intervention treatment, which has the merits of slight wound and better curative effects, becomes the most effective way to treat arterial stenosis. Though the success rate of stent intervention treatment is high, the postoperative In-Stent-Restenosis (ISR, Figure 1) remains a challenge for medical sciences and biomechanical engineering [3].

Stenting, which can restore vessel lumen for arterial stenosis by its mechanical support, may cause the adaptive response of vessels. Studies have shown that ISR is not only associated with the vessel wall damage which includes endothelial denudation by the stent struts during expansion, the damage of elastic layer and smooth muscle, and long-term external forces by stents to the vessels after stenting $[4,5]$, but also closely related to the blood flow patterns within the vessel lesions, as well as other hemodynamic parameters such as wall shear stress (WSS), oscillatory shear index (OSI) [6], particle residence time, disturbed flow, etc. [7]. Taking into account the impacts of mechanical support of stent struts on vessel wall and the vessel response to blood flow, the biomechanics research of stents in arterial stenosis plays an important role in long-term efficacy of treatment after stenting.

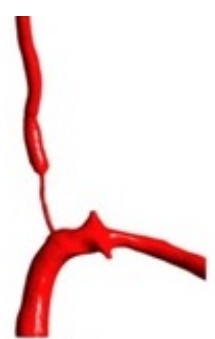

(a)

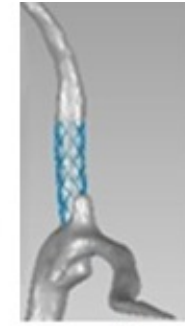

(b)

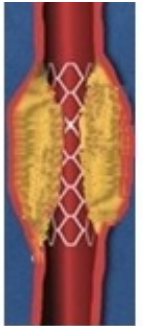

(c)
Figure 1: Arterial stenosis treated with endovascular stent and postoperation In-Stent Stenosis. (a) Arterial stenosis; (b) stent intervention; (c) In-Stent Restenosis

This paper reviews the progress and state-of-the-art situation of biomechanics studies on stenting in arterial stenosis, and analyzes and summarizes the biomechanical factors of ISR. Specifically, from the perspectives of solid mechanics and hemodynamics, the paper elaborates the research progress in recent years on the biomechanical simulation of stenting in arterial stenosis rather than inhibition of restenosis by drugs; therefore, this study does not involve drug-eluting stents.

\section{Solid Mechanics Studies on Post-stenting Procedure}

After the stent intervention for arterial stenosis, the mechanical support by stent recovers the lesion and results in recanalization, but it also leads to a large non-tissue stress and strain in lesion. Stent not only changes the local shape of the blood vessel, but also causes stress concentrations in local vessel wall. Although the flexibility of selfexpanding stent depicts a very good improvement to that of balloonexpanded stent, there is contact stress between vessel and stent which could irritate vessel to cause adaptive response. 
The reaction and remodeling of vessels for stenting includes four periods: thrombosis, inflammation, intimal hyperplasia and vessel remodeling. These all start with the injury of vascular endothelial cells, and the damage of vessel wall resulting from stent leads to endothelial denudation, then prompts the local thrombosis whose severity is closely related to the depth of penetration of the vessels. The inflammation is a self-healing process on the elastic membrane where monocytes and polymorphonuclear leukocytes adhere. Endometrial hyperplasia occurs after the migration of smooth muscle cells into the intima. With the score of poorly differentiated contractile to synthetic transformation, a large number of protein and collagen deposit in the intima and adventitia of vessel, and finally are fiberized. Vessel remodeling resulting from excessive intimal hyperplasia is clinically known as ISR [7].

With the further improvement of imaging technology and image reconstruction techniques, the change of vessel shapes can be observed, while the observations of stress distribution on the vessel can only be performed by means of numerical simulation techniques. Comparing the deformation capacities of stent and vessel, it can be seen that stent structure almost can be considered as a non-deformable material. Vessels, as their non-homogeneous, anisotropic and nonlinear hyper-elastic properties, can have large deformation even without the action of the stent. In addition, friction issues between vessels during the stent expansion procedure increase the difficulty of vessel stress analysis after stenting. To a large extent, another factor that affects the prediction of vessel stress is the estimation of zerostress state and residual stresses in vessel wall $[8,9]$. The interaction between the mechanical support by stent and vessels retraction makes the analysis of vessel stress more complicated [10].

In order to study the possible mechanical factors of vessel damage in the stent expansion process, Rogers et al established the models of balloons, stents and vessels with their interactions, and then numerical simulation analysis was carried out [11]. The results showed that the stress distribution is closely related to the space between the supporting links of stent. Higher stress values are predicted with the higher pressure of balloon, the larger strut space or more flexible balloon. However, the conclusion that larger strut space indicates higher stress is contrary to that of Gu et al., who found that larger strut space would alleviate vessel stress concentration, meanwhile significantly reduce the peak stress [12].

For the purpose of studying the relationship between ISR and vascular stress, Gu et al., built the stent models of PS and S670 which both could cause different rates of restenosis. The results demonstrate that peak stress of vessel induced by PS stent is 2 times of that induced by $S 670$ stent. The rates of ISR caused by PS stent and S670 stent are respectively $31.6 \%$ and $16 \%$. Thus, Gu et al believe that the rate of ISR is associated with peak stress of vessel. Compared with the strut space of PS stent, S670 stent has larger ones, therefore Gu et al., believe that larger strut space tends to reduce vessel stress and the vessel wall damage induced by the mechanical support of stent.

Rogers et al., got the opposite conclusions, which might be related to the model they built. Compared to the model Gu established, there is an additive balloon model in Rogers' study. It is likely suggested that balloon model results in the two different conclusions. Although contrary does exist, these two conclusions are both associated with strut space of stent. It demonstrates that vessel stress caused by the stent is closely related to the strut space of stent. But this needs further investigation to explore what exactly is the relationship between the two. At least, it is envisioned that the larger strut space of stent, the better flexibility of stent, and the relatively smaller stress on the vessel wall induced by stent.

Auricchio et al., built carotid artery model by using image reconstruction techniques, and performed the study of numerical simulation for the stent deployment in patient-specific model [13]. The results show that the bend segments of the vessel are obviously straightened after stenting (Figure 2); obvious deformation occurs at vessel cross-section; peak stress of the vessel wall arises at the stenosis. The phenomenon of vessel straightening and peak stress in vessel stenosis, etc. agree well with the study of $\mathrm{Wu}$ et al who simulated the expansion of stent in straight and curved vessels [14]. The results show that peak stress appears at the stenosis of straight and curved vessels, but curved vessels has larger peak stress which appears at the inside of the bend. It was considered by Wu et al that high stress of vessel led to the increase of risk of ISR.

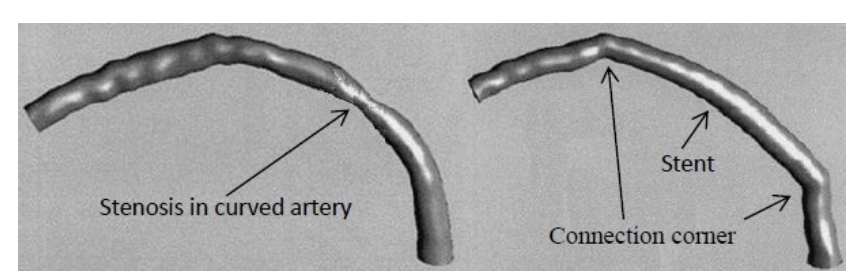

Figure 2: Strengthening effect of stent in curved stenotic artery. Left: curved stenotic artery; Right: Strengthening effect of stented artery.

From the above analysis, it can be seen that stenting brings about the vessel deformation, non-tissue stress and stress concentration in vessel. The adaptive response caused by these eventually leads to ISR. Many factors affect the vessel stress, including the stent strut space, the stent thickness and the bend degree of vessel, etc. However, there is rare numerical simulation about the effect of the form of stent strut on vessel stress. As a result, further study about this issue should be settled in the future.

\section{Hemodynamic study on stented artery}

The compliance of stent cannot be compared with that of normal vessel. The stented vessel has a compliance mismatch with the normal vessels at the two ends of stent. For this reason, stenting causes a significant straightening of vessel, and part of the blood can be folded or bounced back which causes disturbed blood flow [13-18]. Wu et al. performed numerical simulation of solid mechanics of the influence of stent on vessel, but they believed that blood flow mechanics had greater impact on ISR than the local solid mechanics of stent [14]. The mismatch between the stent and vessel might bring about disturbed flow which could cause thrombosis [7]. This is consistent with the viewpoint of Wentzel et al. [17]. Wentzel et al. found that due to the flexibility mismatch between stent and vessel, the stent after deployment caused a connection corner on curved blood vessels and a sudden change of vessel curvature at the end of stent (Figure 2). At the connection corner, the axial inconsistent hardness between hard stent ends and soft vessel induces greater stimuli in the vessel than elsewhere, which is more prone to trigger ISR. In addition, the change of vessel curvature resulted from the obvious straightening of vessel also changes local hemodynamic environment in the vicinity of stent. These might produce disturbed flow which might trigger thrombosis. 
The placement of stent not only affects the whole blood flow pattern in vessel, but also has great impact on blood flow in the vicinity of vessel wall. The stent strut, which has a certain thickness, protrudes into the vessel lumen and forms a stepped shape which is connected with the vessel wall. Thereby the blood flow demonstrates typical forward step flow and backward step flow which may cause flow separation (Figure 3). With a series of adjacent and interactive forward step flow and backward step flow, more complex blood flow patterns are generated [7]. Deng et al. also shared the view that the stent strut has a great impact on intravascular local flow patterns [19]. Too low WSS resulted from the arising of vortex and flow separation in the back of stent strut induces harmful substances to residence too long time in the vicinity of blood vessel wall, thereby the initiation area of ISR is prone to occur at the back of stent strut. With respect to the long residence time of harmful substances, Deng et al. believe that the swirling flow of human aortic arch can wash the vessel wall and reduce the deposition of harmful substances and thus ISR can be alleviated [20]. Deng et al. compared the hemodynamic difference of stent under swirling flow and normal flow. The results show that under the swirling flow, the OSI near the stent strut is significantly reduced and the long residence time of harmful substances is shortened. As a result, based on the principle of swirling flow, Deng et al designed a guide device of swirling flow which could guide the blood flow to arise swirling flow, and the device was optimized by using numerical simulation of hemodynamics [21].

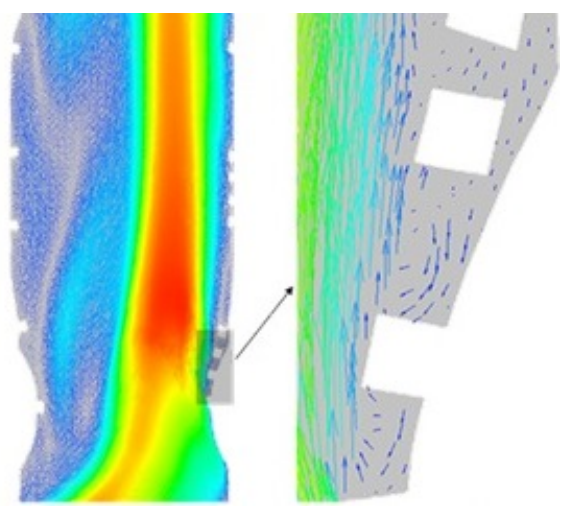

Figure 3: Step flow near the stent strut.

Studies have shown that WSS has key influence on the alignment and morphology of vessel endothelial cells. Under the physiological condition of WSS levels $(>1.5 \mathrm{~Pa})$, endothelial cells grow in the direction of blood flow, which can prevent atherosclerosis. Under the pathological conditions of the WSS levels $(<0.4 \mathrm{~Pa})$, endothelial cells are greatly proliferated and distributes in any portion of the blood vessel with any shape and direction, which can promote atherosclerosis [22,23]. Jiang et al. found that low WSS causes vessel remodeling and the apoptosis of smooth muscle cells [24]. To understand the impact of fluid shear stress gradient on the morphology of endothelial cell, Jiang et al. studied the morphology and arrangement of endothelial cells under different shear stress gradient [25], and found that the direction of endothelial cells does not follow any definite rules and the arrangement of cells varies under different fluid shear stress gradients. For studying the process of ISR, De Santis et al. established a mathematical model to show the influence of stent on WSS [26]. Changes in the shape of the blood vessels cause changes in WSS; by changing the shape of vessel and hence changing the WSS up to a certain threshold, numerical simulations of hemodynamics were performed to predict the remodeling shape of vessel. Only two-dimensional model was simulated by De Santis et al, and the three-dimensional model is of greater significance and it may become a pre-clinical virtual forecasting tool. Chen et al studied the effect of the diameter size of the stent on the WSS. The study reveals that appropriate diameter of stent can increase WSS and reduce OSI, and profits to reduce the adverse effects of blood flow on vessels [27]. Cheng et al. carried out numerical simulations of hemodynamics for stenotic vessel and its stenting treatment [28]. The results show that low WSS and oscillatory shear stress play major roles in ISR. Morlacchi et al put stent into pig's vessel which led to the remodeling of vessel, and then numerical simulation of hemodynamics was carried out based on analyzing Micro-CT image of vessel and building the reconstruction models of vessel and stent [29]. By comparing the experimental and numerical results, Morlacchi et al found that low WSS and high OSI are prone to initiate ISR.

Qiao et al. designed three types of stent with different links (L-stent, V-stent and S-stent) and an idealized stenotic vertebral artery [18]. The deployment procedure for the stent in the stenotic vertebral artery was simulated for solid mechanics analysis. Then, the deformed models were extracted to construct the blood flow domain, and numerical simulations of the hemodynamics in these models were performed using the finite element method (Figure 4). From the viewpoint of the combination of solid mechanics and hemodynamics, the S-stent has better therapeutic effects because of its lower potential for inducing ISR and its better prospects in clinical applications compared with the L-stent and V-stent.
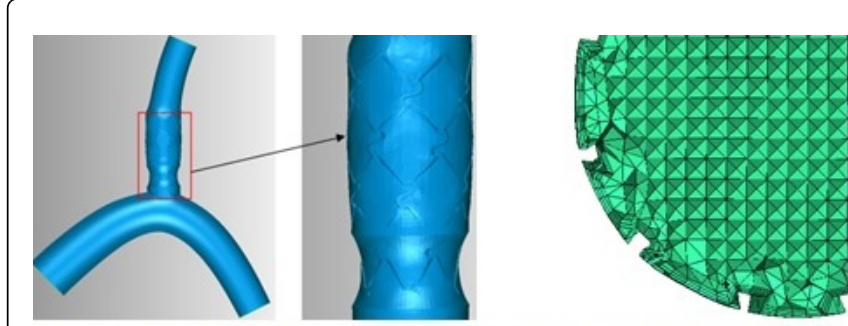

Figure 4: Blood flow domain extracted from the deformed stented vertebral artery (Left) and the mesh of fluid field (Right).

From the above analyses, it can be seen that the deformation of vessel by stenting not only affects the flow pattern in the region closed to the vessel wall, but also has an effect on the whole flow pattern in vessel. Thus the changes of WSS, OSI and regions of stagnant flow and other hydrodynamic parameters can lead to ISR. Currently, researchers often built the needed vessel models based on medical images by using three-dimensional reconstruction techniques, and then study the hemodynamic differences of stenotic vessel before and after stenting. To involve the detailed effect of stent on the flow, it is needed to reconstruct the stent by using the scanned images with high resolution, such as Micro-CT images, etc. This kind of way is time consuming and expensive. However, studies on the effect of arterial stenosis and its stent intervention by using numerical simulation method combining solid mechanics and fluid dynamics, are rarely reported. Among these, issues as how to easily import the deformed model after solid mechanics simulation into flow field for hemodynamics simulation and then numerically simulate the fluidstructure interaction of stented artery based the deformed vessel wall 
and stent strut with residual stresses is a critical matter which play the key bridging role.

\section{Perspectives}

ISR occurred after stent intervention treatment of arterial stenosis is a problem which cannot be neglected. ISR has a relationship with the biomechanical factors after stent expansion. This paper reviewed numerical simulation studies of the solid mechanics and hemodynamics for the stenotic vessel and its stent intervention treatment. It can be seen that the adaptive response of vessel induced by high peak stress, stress concentration, WSS, OSI, region of flow stagnation and other biomechanical factors eventually leads to ISR.

With regard to the numerical studies of biomechanics of stented stenotic artery, there are three key issues: First, the segmentation of vessel wall and plaque (Figure 5), and the assignment of different material properties for them, especially for those complex plaque with multiple components [30,31]; Second, the reconstruction of blood flow domain based on the deformed stented stenotic artery (Figure 4); And third, the residual stresses assignment and the fluid-structure interaction analysis of the deformed model.

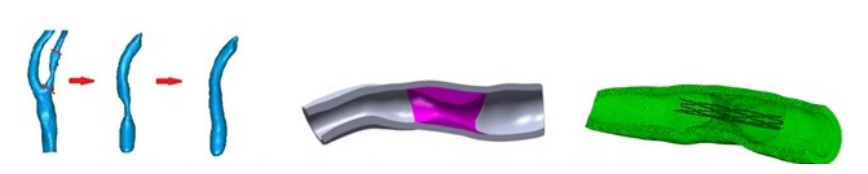

Figure 5: Segmentation of blood vessel wall and plaque (Left and Middle), and their assembly with stent (Right).

With advances in imaging technology and image reconstruction techniques, the time of rebuilding patient-specific models can be further reduced and the shapes of models can be more precisely expressed. Material properties of blood vessels will also be more accurately described, and the intima, media and adventitia can be assigned the anisotropic and viscoelastic hyperelastic properties which can be changed with the change of specific location [29]. While nonlaminar and non-Newtonian blood flow can be used for numerical simulations of hemodynamics to make the situation more realistic.

For a comprehensive analyses of structural mechanics and fluid mechanics problems after stenting for stenotic arteries, the flow analysis, even fluid-structure interaction analysis are needed based on the deformed vessel-stent model with contacting action. For this reason, the interaction between vessel and stent and hemodynamic situation after stent intervention treatment should be investigated in depth to provide a scientific basis of the selection and design of stent, the surgical planning of stent intervention and so on.

\section{Acknowledgements}

This work was supported by National Natural Science Foundation of China (81171107).

\section{References}

1. Jashari F, Ibrahimi P, Nicoll R, Bajraktari G, Wester P, et al. (2013) Coronary and carotid atherosclerosis: similarities and differences. Atherosclerosis 227: 193-200.
2. Albuquerque FC, Fiorella D, Han P, Spetzler RF, McDougall CG (2003) A reappraisal of angioplasty and stenting for the treatment of vertebral origin stenosis. Neurosurgery 53: 607-614.

3. Alfonso F, Byrne RA, Rivero F, Kastrati A (2014) Current treatment of in-stent restenosis. J Am Coll Cardiol 63: 2659-2673.

4. Colombo A, Stankovic G, Moses JW (2002) Selection of coronary stents. J Am Coll Cardiol 40: 1021-1033.

5. Phillips PS, Alfonso F, Segovia J, Goicolea J, Hernandez R, et al. (1997) Effects of Palmaz-Schatz stents on angled coronary arteries. Am J Cardiol 79: 191-193.

6. Ku DN, Giddens DP, Zarins CK, Glagov S (1985) Pulsatile flow and atherosclerosis in the human carotid bifurcation. Positive correlation between plaque location and low oscillating shear stress. Arteriosclerosis 5: 293-302.

7. Chiu JJ, Chien S (2011) Effects of disturbed flow on vascular endothelium: pathophysiological basis and clinical perspectives. Physiol Rev 91: 327-387.

8. Han H, Lam DC, Huang W (2012) Effect of danshen on the zero-stress state of rat's abdominal aorta. Mol Cell Biomech 9: 295-307.

9. Haghighipour N, Tafazzoli-Shadpour M, Avolio A (2010) Residual stress distribution in a lamellar model of the arterial wall. J Med Eng Technol 34: 422-428.

10. Fung Y C (1991) What are the residual-stresses doing in our bloodvessels. ANNALS OF BIOMEDICAL ENGINEERING 19: 237-249.

11. Rogers C, Tseng DY, Squire JC, Edelman ER (1999) Balloon-artery interactions during stent placement: a finite element analysis approach to pressure, compliance, and stent design as contributors to vascular injury. Circ Res 84: 378-383.

12. Gu L X, Zhao S J, Muttyam A K, Hammel JM (2010) The relation between the arterial stress and restenosis rate after coronary stenting. JOURNAL OF MEDICAL DEVICES-TRANSACTIONS OF THE ASME 4: 1-7.

13. Auricchio F, Conti M, De Beule M, De Santis G, Verhegghe B (2011) Carotid artery stenting simulation: from patient-specific images to finite element analysis. Med Eng Phys 33: 281-289.

14. Wu W, Wang WQ, Yang DZ, Qi M (2007) Stent expansion in curved vessel and their interactions: a finite element analysis. J Biomech 40: 2580-2585.

15. Selvarasu NK, Tafti DK, Vlachos PP (2011) Hydrodynamic effects of compliance mismatch in stented arteries. J Biomech Eng 133: 021008.

16. Mortier P, Holzapfel GA, De Beule M, Van Loo D, Taeymans Y, et al. (2010) A novel simulation strategy for stent insertion and deployment in curved coronary bifurcations: comparison of three drug-eluting stents. Ann Biomed Eng 38: 88-99.

17. Wentzel JJ, M Whelan D, Van Der Giessen WJ, Van Beusekom H MM, Andhyiswara I et al. (2000) Coronary stent implantation changes 3-D vessel geometry and $3-\mathrm{D}$ shear stress distribution. JOURNAL OF BIOMECHANICS 33: 1287-1295.

18. Qiao A, Zhang Z (2014) Numerical simulation of vertebral artery stenosis treated with different stents. J Biomech Eng 136.

19. Sun A, Fan Y, Deng X (2012) Intentionally induced swirling flow may improve the hemodynamic performance of coronary bifurcation stenting. Catheter Cardiovasc Interv 79: 371-377.

20. Chen Z, Fan Y, Deng X, Xu Z (2011) A new way to reduce flow disturbance in endovascular stents: a numerical study. Artif Organs 35: 392-397.

21. Teng X, Deng X (2010) [Optimization of a helical flow inducer of endovascular stent based on the principle of swirling flow in arterial system]. Sheng Wu Yi Xue Gong Cheng Xue Za Zhi 27: 429-434.

22. Balossino R, Gervaso F, Migliavacca F, Dubini G (2008) Effects of different stent designs on local hemodynamics in stented arteries. J Biomech 41: 1053-1061.

23. Martin D, Zaman A, Hacker J, Mendelow D, Birchall D (2009) Analysis of haemodynamic factors involved in carotid atherosclerosis using computational fluid dynamics. Br J Radiol 82 Spec No 1: S33-38. 
Citation: Qiao A, Fu Y, Zhang Z (2015) Research of Numerical Simulation of Biomechanics on In-stent-restenosis. J Vasc Med Surg 3: 181. doi:

24. Guo ZY, Yan ZQ, Bai L, Zhang ML, Jiang ZL (2008) Flow shear stres affects macromolecular accumulation through modulation of internal elastic lamina fenestrae. Clin Biomech (Bristol, Avon) 23 Suppl 1: S104-111.

25. Wang YH, Yan ZQ, Shen BR, Zhang L, Zhang P, et al. (2009) Vascular smooth muscle cells promote endothelial cell adhesion via microtubule dynamics and activation of paxillin and the extracellular signal-regulated kinase (ERK) pathway in a co-culture system. Eur J Cell Biol 88: 701-709.

26. De Santis G, Mortier P, De Beule M, Segers P, Verdonck P, et al. (2011) Simulation of wall shear stress-driven in-stent restenosis. JOURNAL OF THE ROYAL SOCIETY 1: 365-373.

27. Chen HY, Hermiller J, Sinha AK, Sturek M, Zhu L, et al. (2009) Effects of stent sizing on endothelial and vessel wall stress: potential mechanisms for in-stent restenosis. J Appl Physiol 106: 1686-1691.
28. Cheng J, Ni Z (2010) A numerical study on the wall shear stress of stented artery model. 2010 International Conference on Mechatronics and Automation (ICMA).

29. Morlacchi S, Keller B, Arcangeli P, Balzan M, Migliavacca F, et al. (2011) Hemodynamics and in-stent restenosis: micro-CT images, histology, and computer simulations. Ann Biomed Eng 39: 2615-2626.

30. Fu Y, Qiao A, Jin L (2015) The influence of hemodynamics on the ulceration plaques of carotid artery stenosis. J. MECH. MED. BIOL 15: 1-14.

31. Lu J, Duan W, Qiao A (2015) Finite element analysis of mechanics of neovessels with intraplaque hemorrhage in carotid atherosclerosis. Biomed Eng Online 14 Suppl 1: S3. 